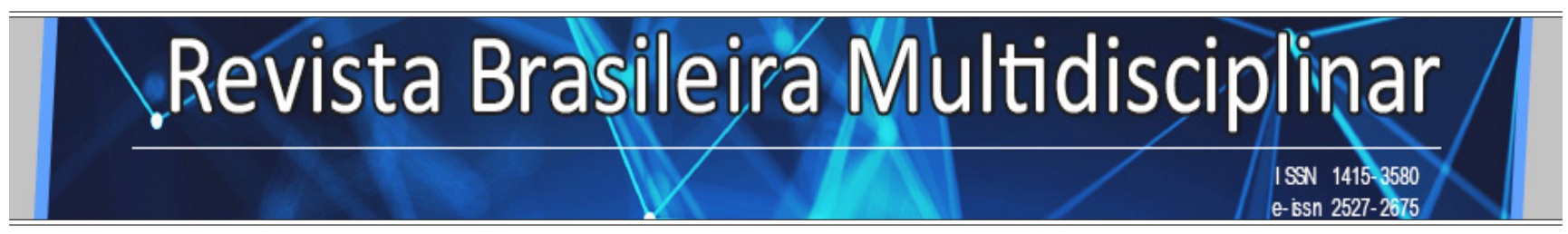

http://revistarebram.com/index.php/revistauniara

\title{
Práticas EDUCATIVAS do ENFERMEIRO NO CONTEXTO DA SAÚDE ESCOLAR: REVISÃo INTEGRATIVA DA LITERATURA
}

Luciana dos Santos Joia*; Adriana Aparecida Mendes ${ }^{* *}$; Mariana Firmino Daré ${ }^{* *}$; Luciana Mara Monti Fonseca ${ }^{* * *}$; Aline Natalia Domingues ${ }^{\star * \star *}$.

* Graduação em Enfermagem Universidade de Araraquara UNIARA.

** Doutora em Ciência pela Escola de Enfermagem de Ribeirão Preto da Universidade de São Paulo - EERP/USP.

*** Doutorado em Enfermagem em Saúde Pública pela Universidade de São Paulo.

**** Doutoranda pelo Programa de Pós-Graduação em Enfermagem em Saúde Pública da Escola de Enfermagem de Ribeirão Preto da Universidade de São Paulo.

*Autor para correspondência e-mail: alinenataliadomingues@gmail.com

\section{Palavras-chave}

\section{Enfermagem}

Serviços de saúde escolar

Educação em saúde

Promoção em saúde

\section{KEYWORDS}

Nursing

School health services

Health education

Health promotion

\section{RESUMO}

Este estudo teve por objetivo conhecer a prática do enfermeiro na educação em saúde do escolar através de uma revisão integrativa de literatura. A busca dos artigos científicos foi realizada nas bases de dados eletrônicas: Literatura Latino-Americana e do Caribe em Ciências da Saúde (LILACS), Cumulative Index to Nursing and Allied Health Literature (CINHAL) e Medical Literature Analysis and Retrieval System Online (MEDLINE), com critérios de inclusão e exclusão pré-definidos. A busca resultou na seleção de oito estudos, que responderam à pergunta norteadora, com predomínio de temas relacionados à prevenção de doenças cardíacas e obesidade. Conclui-se que esta área ainda é incipiente em termos de publicações sobre a prática educativa do enfermeiro nas escolas, atuando diretamente com crianças e adolescentes, tendo em vista a importância da atuação enfermeiro nas escolas para a promoção e prevenção em saúde.

\section{EDUCATIONAL PRACTICES OF NURSES IN THE SCHOOL HEALTH CONTEXT: INTEGRATIVE LITERATURE REVIEW}

This study aimed to know the nurse's practice in school health education through an integrative literature review. The search for scientific articles was carried out in the electronic databases: Latin American and Caribbean Literature in Health Sciences (LILACS), Cumulative Index to Nursing and Allied Health Literature (CINHAL) and Medical Literature Analysis and Retrieval System Online (MEDLINE), with pre-defined inclusion and exclusion criteria. The search resulted in the selection of eight studies, which answered the guiding question, with a predominance of topics related to the prevention of heart disease and obesity. It is concluded that this area is still incipient in terms of publications on the educational practice of nurses in schools, working directly with children and adolescents, in view of the importance of nurses in schools for health promotion and prevention. 


\section{INTRODUÇão}

A Associação Nacional de Enfermeiras Escolares, dos Estados Unidos, define a enfermagem escolar como uma prática especializada que viabiliza a saúde, a educação e a satisfação durante a vida, favorecendo o desenvolvimento, proporcionando saúde e autoconfiança, atuando na prevenção e promoção das patologias, gerenciando os casos e colaborando para o cuidado e autopercepção do aluno e sua família (AMERICAN ACADEMY OF PEDIATRICS, 2008).

A enfermeira escolar é uma prática de saúde existente em vários países, como Alemanha, Suíça, Reino Unido e Espanha, onde o enfermeiro possui as seguintes funções: assistencial (prestando cuidados integrais aos alunos, familiares e funcionários das escolas), docente (realizando atividades educativas), investigativa (desenvolvendo trabalhos científicos) e administrativa (planejando, organizando e realizando a gestão dos recursos e atividades) (FERNANDINO, 2016).

No Brasil, a inserção de uma estratégia que abordasse a saúde surgiu na metade do século XIX e era destinada a classe social elevada, chegando às classes sociais menos favorecidas no início do século XX, devido à necessidade de combater epidemias no país (FERREIRA et al., 2016).

A educação em saúde nas escolas trata-se de um trabalho que depende de profissionais com habilidades e competências para orientar os indivíduos na promoção, prevenção de doenças e agravos à saúde e na sua restauração (COSTA; FIGUEIREDO; RIBEIRO, 2013).

A sala de aula deve ser um espaço dinâmico de autonomia de vida, contribuindo para o desenvolvimento de habilidades que resultem na conscientização de que as pessoas respeitem e convivam com as diferenças existentes entre elas (GIJSEN; KAISER, 2013).

A Portaria 687/2006 do Ministério da Saúde dispõe sobre a Política Nacional de Promoção da Saúde (PNPS) inserindo ações de promoção de saúde em todos os locais onde se desenvolvem atividades de cuidado humano em unidades de saúde e espaços coletivos (COSTA et al., 2013).

O Programa Saúde na Escola (PSE), criado através da união do Ministério da Saúde e do Ministério da Educação, em 2007, pelo Decreto n ${ }^{\circ}$ 6.286, mediante a criação de políticas intersetoriais, pelo governo federal, visando promover a melhoria da qualidade de vida dos cidadãos brasileiros. As políticas do programa saúde nas escolas são direcionadas às crianças, adolescentes, jovens e adultos que frequentam a educação pública, através de creches, pré-escolas, escolas de ensino fundamental e médio e educação para jovens e adultos (EJA) (BRASIL, 2015).

A coordenação do programa saúde nas escolas acontece através dos Grupos de Trabalho Inter setoriais por meio de uma gestão compartilhada, de forma que a organização e a efetivação das intervenções aconteçam atendendo às demandas do território. Desse modo, ocorre uma relação de intercâmbio mútuo de conhecimentos entre a Saúde, a Educação e a população (BRASIL, 2015).

De acordo com o Programa Saúde na Escola, as equipes da Estratégia de Saúde da Família podem atuar em conjunto com a Educação Básica para a realização das atividades de educação, promoção e prevenção em saúde no ambiente escolar. As ações de promoção, prevenção e educação em saúde, através da avaliação da equipe multidisciplinar, colaboram com a diminuição nas taxas de morbidade e mortalidade, visto que possibilitam o reconhecimento e a intervenção nos fatores de risco das patologias (BRASIL, 2015).

A atuação do enfermeiro poderá ser realizada através do Programa Saúde na Escola, Decreto 6.286/2007 que surgiu como uma política intersetorial entre Ministério da Saúde e ministério da Educação (COSTA et al., 2013).

Na escola esse vínculo entre saúde e educação possibilita o direcionamento ao atendimento do escolar na atenção básica ou especializada, contribuindo para as ações de saúde, por meio da educação em saúde (CASEMIRO; FONSECA; SECCO, 2014).

Desta forma, o enfermeiro tem o compromisso com a saúde da população, bem como, o conhecimento 
em nível maior sobre saúde a sua atuação como educador em saúde com escolares, disseminando informações de promoção da saúde e auxiliando na prevenção de estilo de vida desfavorável à saúde (COSTA et al., 2013).

O enfermeiro está apto para exercer a educação em saúde, pois uma de suas funções é promover a formação do conhecimento em saúde individual e coletiva respeitando em cada indivíduo seus valores, princípios e crenças (COSTA et al., 2013; GIJSEN; KAISER, 2013). Deste modo, a atuação do enfermeiro no desenvolvimento das ações e práticas relacionadas à educação em saúde no ambiente escolar é de suma importância, além disso, é necessário atentar-se ao surgimento de novas Políticas Nacionais de Saúde e o cumprimento de sua função educativa na sociedade (RASCHE; SANTOS, 2013).

Diante do exposto, este estudo teve como objetivo conhecer as práticas educativas do enfermeiro no contexto da saúde do escolar através de uma revisão integrativa da literatura.

\section{Metodologia}

Esta pesquisa foi fundamentada no percurso metodológico de uma revisão integrativa da literatura. Este método consiste em sintetizar e avaliar as evidências científicas disponíveis na literatura, com o intuito de sistematizar estratégias para melhor compreensão sobre determinado problema de pesquisa. As revisões são importantes para a construção do conhecimento científico porque permitem o surgimento de novas teorias, identificação de lacunas e oportunidades de ascensão de apoio à investigação (MENDES; SILVEIRA; GALVÃO, 2008).

Para operacionalização da revisão, foram realizadas seis etapas distintas, descritas a seguir: 1. Seleção das questões para revisão; 2 . Estabelecimento de critérios para inclusão de estudos e busca na literatura; 3. Representação das características dos estudos revisados; 4. Análise dos estudos utilizando instrumento específico; 5. Interpretação dos resultados e 6. Apresentação dos resultados e síntese do conhecimento (GANONG, 1987).

Para a etapa 1, elaborou a seguinte pergunta norteadora: Quais são as práticas educativas desenvolvida por enfermeiros com crianças e adolescentes no contexto da saúde na escola?

A seleção dos artigos para a revisão ocorreu através da busca nas seguintes bases de dados: Literatura Latino-Americana de Ciências da Saúde (LILACS), Medical Literature Analysis and Retrieval System onLine (MEDLINE) e Cumulative Index to Nursing and Allied Health Literature (CINHAL), através da consulta dos números das revistas disponíveis online.

Em relação a terminologia utilizada foram selecionados os Descritores em Ciências da Saúde (DeCS/ Bireme) e no Medical Subject Headings (MeSH/PubMed), respectivamente: serviços de saúde escolar, enfermagem, educação em saúde, promoção de saúde, school health services, nursing, health education e health promotion, separadas pelo operador booleano and. Dessa forma, buscou-se ampliar o âmbito da pesquisa, utilizando estratégias de buscas, apresentadas no Quadro 1, com o intuito de minimizar possíveis vieses nessa etapa do processo de elaboração desta revisão.

Como critérios de inclusão nesta pesquisa, foram estabelecidos os seguintes parâmetros: artigos indexados nas bases de dados supracitadas, disponíveis on-line na íntegra, nos idiomas inglês, espanhol e português, não limitando período/data de publicação. Como critérios de exclusão, optou-se por excluir publicações secundárias como: artigos de revisão, livros, teses, dissertações. O desenvolvimento do estudo foi efetivado nos meses de julho a outubro de 2019.

Para a análise crítica dos artigos, os dados foram organizados, através da utilização do instrumento URSI (2005), que contempla os seguintes itens: identificação do artigo original, características metodológicas do estudo, avaliação do rigor metodológico, das intervenções mensuradas e dos resultados encontrados.

Os estudos foram classificados, em relação ao seu nível de evidência segundo a categorização da Agency 
for Healthcare Research and Quality (AGENCY FOR HEALTHCARE RESEARCH AND QUALITY, 2017) dos Estados Unidos da América, a saber: nível 1- metanálise de múltiplos estudos controlados; nível 2estudo individual com desenho experimental; nível 3- estudo com desenho quase-experimental como estudo sem randomização com grupo único pré e pós-teste, séries temporais ou caso-controle; nível 4estudo com desenho não-experimental como pesquisa descritiva correlacional e qualitativa ou estudos de caso; nível 5- relatório de casos ou dado obtido de forma sistemática, de qualidade verificável ou dados de avaliação de programas e nível 6- opinião de autoridades respeitáveis baseada na competência clínica ou opinião de comitês de especialistas, incluindo interpretações de informações não baseadas em pesquisas; opiniões reguladoras ou legais.

Quadro 1 - Estratégia de busca pelos descritores controlados. Araraquara, SP, 2020.

\begin{tabular}{|c|c|}
\hline Base de dados & Estratégia de busca (descritores + operador booleano (and) \\
\hline \multirow{2}{*}{ CINAHL } & $\begin{array}{l}\text { Estratégia 1: School Health Services and nursing and Health Education } \\
\text { Estratégia 2: School Health Services and nursing and Health Promotion }\end{array}$ \\
\hline \multirow{2}{*}{ LILACS } & $\begin{array}{l}\text { Estratégia 1: Serviços de saúde escolar and enfermagem and educação em saúde } \\
\text { Estratégia 2: Serviços de saúde escolar and enfermagem and promoção em saúde }\end{array}$ \\
\hline MEDLINE & $\begin{array}{l}\text { Estratégia 1: School Health Services and nursing and Health Education } \\
\text { Estratégia 2: School Health Services and nursing and Health Promotion }\end{array}$ \\
\hline
\end{tabular}

Fonte: Elaborado pelos autores.

\section{RESUltados}

Através da busca nas bases de dados estabelecidas obteve-se um total de 368 publicações. A síntese do processo de seleção dos artigos encontra-se na Figura 1. De acordo com critérios de inclusão previamente determinados, oito artigos foram selecionados para a presente revisão.

Na tabela 1, encontra-se informações referentes ao ano de publicação, idioma, tipo de abordagem da pesquisa, regiões das intervenções, temáticas abordadas, características e público alvo. A existência de poucas publicações nos últimos 5 anos, é a razão de não ter limitado o ano de publicação.

Os estudos selecionados para esta revisão estão apresentados na Quadro 2 e identificados através do título seguido por número ( 1 a 8), com o intuito de facilitar a análise. Os estudos são classificados de acordo com o periódico, base de dados, autores e nível de evidência. 
Figura 1 - Fluxograma representativo do processo de seleção dos artigos para revisão integrativa da literatura. Araraquara, SP, 2020.

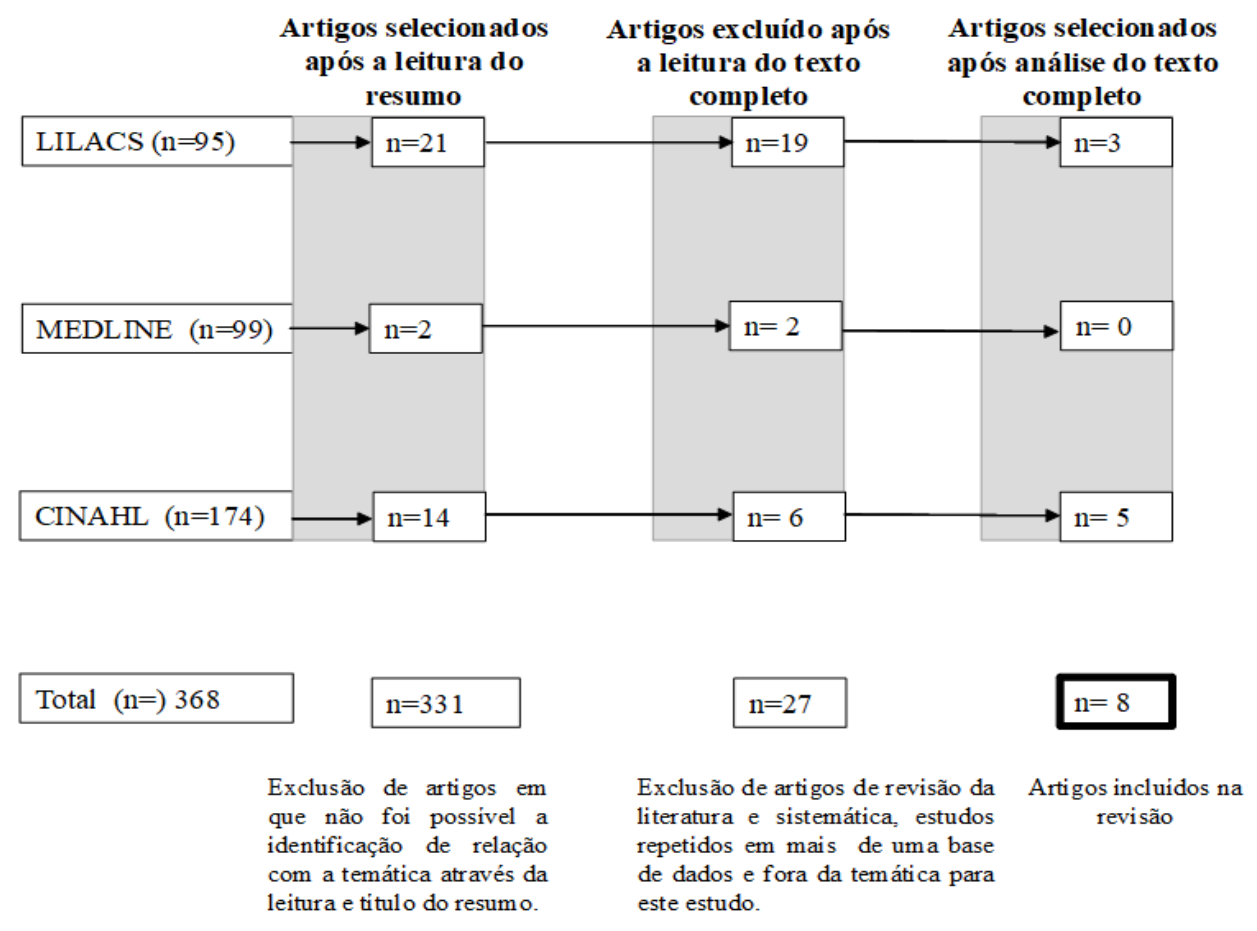

Fonte: Elaborado pelos autores. 
Tabela 1- Caracterização dos estudos quanto ao ano, idioma, abordagem e local de intervenção segundo frequência e porcentagem. Araraquara. SP, 2020.

\begin{tabular}{|c|c|c|c|}
\hline Variável & & $\mathbf{N}$ & $\%$ \\
\hline \multirow{3}{*}{ Ano de publicação } & $1997-1999$ & & $12,5 \%$ \\
\hline & $2000-2009$ & $\begin{array}{l}1 \\
4\end{array}$ & $50 \%$ \\
\hline & $2010-2018$ & 3 & $37,5 \%$ \\
\hline \multirow{3}{*}{ Idioma } & Português & 3 & $37,5 \%$ \\
\hline & Inglês & 5 & $62,5 \%$ \\
\hline & & 4 & $50 \%$ \\
\hline \multirow{4}{*}{$\begin{array}{l}\text { Tipo de abordagem da } \\
\text { pesquisa }\end{array}$} & Qualitativa - pesquisa ação & 2 & $25 \%$ \\
\hline & Qualitativa - relato de experiência & 1 & $12-0 /$ \\
\hline & Pesquisa quase-experimental & 1 & $12,5 \%$ \\
\hline & & 1 & $12,5 \%$ \\
\hline \multirow{4}{*}{ Regiões das intervenções } & Brasil (Ceará, Pernambuco e São Paulo) & 3 & $37,5 \%$ \\
\hline & Estados Unidos & & \\
\hline & Canadá & 4 & $50 \%$ \\
\hline & & 1 & $\begin{array}{l}12,5 \% \\
12,5 \%\end{array}$ \\
\hline \multirow{10}{*}{ Temática das intervenções } & & 1 & $12,5 \%$ \\
\hline & Violência & 1 & \\
\hline & Desnutrição infantil & 1 & $12,5 \%$ \\
\hline & Sexualidade e DST/HIV/AIDS & & \\
\hline & Prevenção de queimaduras & 1 & $12,5 \%$ \\
\hline & Prevenção de doenças cardíacas / obesidade & 2 & \\
\hline & Prevenção da asma & 1 & $25 \%$ \\
\hline & Avaliação do crescimento / obesidade & & $12,5 \%$ \\
\hline & & 1 & \\
\hline & & & $12,5 \%$ \\
\hline \multirow{2}{*}{$\begin{array}{l}\text { Características das } \\
\text { intervenções }\end{array}$} & Atividade educativa & 5 & $62,5 \%$ \\
\hline & Programa educativo & 3 & $37,5 \%$ \\
\hline \multirow{2}{*}{ Público alvo das intervenções } & Adolescentes & 2 & $25 \%$ \\
\hline & Crianças & 6 & $75 \%$ \\
\hline
\end{tabular}

Fonte: Elaborado pelos autores. 
Quadro 2 - Distribuição dos estudos, segundo títulos, base de dados, autores e nível de evidência. Araraquara, SP, 2020.

\begin{tabular}{|c|c|c|c|c|}
\hline TÍTULO & $\begin{array}{l}\text { BASE DE } \\
\text { DADOS }\end{array}$ & AUTOR(ES) & OBJETIVO & $\begin{array}{l}\text { Nível de } \\
\text { evidência }\end{array}$ \\
\hline $\begin{array}{l}\text { A1. Intervenção educativa sobre violência com } \\
\text { adolescentes: possibilidade para a enfermagem no } \\
\text { contexto escolar }\end{array}$ & LILACS & $\begin{array}{l}\text { BRANDÃO } \\
\text { NETO W.; } \\
\text { et al. }\end{array}$ & $\begin{array}{l}\text { Realizar uma intervenção educativa com ado- } \\
\text { lescentes sobre a violência no contexto esco- } \\
\text { lar, utilizando a metodologia de Círculos de } \\
\text { Cultura. }\end{array}$ & Nível 4 \\
\hline $\begin{array}{l}\text { A2. Comunicação Educativa do enfermeiro na } \\
\text { promoção da saúde sexual do escolar }\end{array}$ & LILACS & $\begin{array}{l}\text { OLIVEIRA, } \\
\text { M.A.F.C.; } \\
\text { BUENO, } \\
\text { S.M.V. }\end{array}$ & $\begin{array}{l}\text { Identificar os reais problemas dos jovens es- } \\
\text { colares. Planejar, executar e avaliar um pro- } \\
\text { grama educativo voltado para as dificuldades } \\
\text { encontradas, em ação conjunta com os alunos } \\
\text { trabalhados. }\end{array}$ & Nível 4 \\
\hline $\begin{array}{l}\text { A3. Educação em saúde na escola: estratégia em } \\
\text { enfermagem na prevenção de desnutrição infantil }\end{array}$ & LILACS & $\begin{array}{l}\text { VASCON- } \\
\text { CELOS, } \\
\text { V.M.; et al. }\end{array}$ & $\begin{array}{l}\text { Identificar a percepção das crianças sobre ali- } \\
\text { mentação saudável, investigar parâmetros de } \\
\text { desnutrição infantil na escola e desenvolver } \\
\text { estratégias de educação em saúde para serem } \\
\text { utilizadas na escola como medida preventiva } \\
\text { da desnutrição infantil. }\end{array}$ & Nível 4 \\
\hline $\begin{array}{l}\text { A4. A Practitioner-Based Asthma Intervention } \\
\text { Program with African American Inner-City School } \\
\text { Children }\end{array}$ & CINAHL & $\begin{array}{l}\text { FRIEDRICH, } \\
\text { B.V.; PIG- } \\
\text { OTT, T. }\end{array}$ & $\begin{array}{l}\text { Discutir os resultados de um programa de in- } \\
\text { tervenção baseado na escola sobre os resulta- } \\
\text { dos psicossociais e de saúde de crianças com } \\
\text { asma. }\end{array}$ & Nível 5 \\
\hline $\begin{array}{l}\text { A5. A Growth Status Measurement Pilot in Four } \\
\text { Calgary Area Schools: Perceptions of Grade } 5 \text { Stu- } \\
\text { dents and Their Parents }\end{array}$ & CINAHL & $\begin{array}{l}\text { JOHNSTON, } \\
\text { J.C.T.; et al. }\end{array}$ & $\begin{array}{l}\text { Projeto piloto: criar um modelo para medição } \\
\text { de crescimento em alunos da } 5^{\mathrm{a}} \text { série na área } \\
\text { de Calgary e para explorar as percepções de } \\
\text { crianças e seus pais para a triagem processo } \\
\text { após a sua implementação. }\end{array}$ & Nível 4 \\
\hline $\begin{array}{l}\text { A6. Nursing students practice primary fire preven- } \\
\text { tion }\end{array}$ & CINAHL & $\begin{array}{l}\text { LEHNA, C.; } \\
\text { et al. }\end{array}$ & $\begin{array}{l}\text { Avaliar o programa de segurança contra incên- } \\
\text { dio doméstico padronizado e interativo, para } \\
\text { alunos do ensino fundamental. }\end{array}$ & Nível 4 \\
\hline $\begin{array}{l}\text { A7. L.I.F.E.: A School-Based Heart-Health Scree- } \\
\text { ning and Intervention Program }\end{array}$ & CINAHL & $\begin{array}{l}\text { NORTHRUP, } \\
\text { K.L.; et al. }\end{array}$ & $\begin{array}{l}\text { Identificar fatores de risco cardiovascula- } \\
\text { res nos estudantes e suas famílias e fornecer } \\
\text { aconselhamento, educação, e oportunidades } \\
\text { para mudar rotinas de estilo de vida que con- } \\
\text { tribuem para esses fatores de risco. }\end{array}$ & Nível 5 \\
\hline $\begin{array}{l}\text { A8. A School-Based Intervention to Teach Third } \\
\text { Grade Children about the Prevention of Heart } \\
\text { Disease }\end{array}$ & CINAHL & $\begin{array}{l}\text { SKYBO, T.; } \\
\text { WENGER, } \\
\text { N.R. }\end{array}$ & $\begin{array}{l}\text { Comparar programa educativo baseado na es- } \\
\text { cola, Heart Power, para o padrão currículo de } \\
\text { educação em saúde. }\end{array}$ & Nível 3 \\
\hline
\end{tabular}

Fonte: Elaborado pelos autores.

\section{DISCUSSÃo}

O número reduzido de artigos encontrados refere-se aos poucos artigos relacionados à atuação direta do enfermeiro nas escolas. Houve predominância de estudos de abordagem qualitativa, com nível de evidência 4 e intervenções de características educativas e voltadas às crianças. As temáticas encontradas foram diversas, destacando-se a prevenção de doenças cardíacas e obesidade.

A predominância de estudos publicados na língua inglesa deve-se ao fator de ser o idioma amplamente 
utilizado pelo mundo, com maior índice de publicações científicas (DRUBIN; KELLOGG, 2012). Além disso, outro fator deve-se que a saúde escolar é amplamente reconhecida e atuante o papel do enfermeiro em escolas da América do Norte e Europa.

Os autores Skybo e Wenger (2002), Northrup et al. (2008) e Johnston et al. (2011), referem-se nos artigos a prevenção de obesidade relacionadas a diminuição de doenças cardiovasculares ou avaliação do crescimento para sua detecção. Em relação a importância da temática, mundialmente a obesidade ocorre em crianças e adolescentes, somando aproximadamente 41 milhões em 2016. Crianças obesas ou com sobrepeso podem adquirir, na vida adulta, doenças cardiovasculares e diabetes, tornando o excesso peso tão prejudicial à saúde quanto a desnutrição (WORLD HEALTH ORGANIZATION, 2017).

Vasconcelos et al. (2008), menciona em seu artigo, a desnutrição infantil. Encontramos a relevância do tema no fator da fome no mundo estar elevando-se mais uma vez, tendo atingido em 2017 cerca de 821 milhões de pessoas subnutridas, resultando em 10,9\% da população mundial. Este número deve-se, principalmente, a instabilidade em regiões conflituosas, condições climáticas adversas e desaceleração econômica (ONU, 2018).

A prevenção da asma nas escolas é o assunto exposto por Friedrich e Pigott (2005), merece destaque por ser considerada a doença crônica respiratória mais comum em crianças, sendo mais grave nos países pobres, já em países desenvolvidos é a causa evitável que mais ocasiona internações hospitalares. Mundialmente ela acomete por volta de 334 milhões de pessoas de todas as idades (ASSOCIAÇÃO LATINO AMERICANA DE TÓRAX, 2017).

A violência no âmbito escolar é apresentada no estudo de Brandão Neto et al. (2014). A violência interpessoal ocasiona problemas sociais e psicológicos em suas vítimas. Relacionando-se com uso inadequado de álcool e drogas, tabagismo, relação sexual desprotegida e, em casos extremos, suicídio. Podendo adquirir doenças cardíacas e infecções sexualmente transmissíveis (WORLD HEALTH ORGANIZATION, 2014).

O estudo de Oliveira e Bueno (1997) apontam a importância da educação na saúde sexual. A puberdade está associada a autossuficiência nas escolhas e nos sentimentos, relacionado à aquisição de novas competências e a experiência sexual, sujeitando os adolescentes a agressões e condutas perigosas, como envolvimento com álcool ou drogas e predisposição às infecções sexualmente transmissíveis ou gestação não planejada. Também podem ocorrer o surgimento de patologias envolvendo a saúde mental (ORGANIZAÇÃO PAN-AMERICANA DA SAÚDE, 2017; BRASIL, 2017).

Lehna et al. (2013), aborda a questão da prevenção de queimaduras. Em 2004, 310.000 pessoas foram a óbito devido a queimaduras, sendo que 30\% delas eram menores de 20 anos. A $11^{\text {a }}$ causa de óbitos em crianças de 1 a 9 anos estão relacionadas a queimaduras pôr fogo (WORLD HEALTH ORGANIZATION, 2008).

As temáticas do PSE, preconizadas pelo Ministério da Saúde, para crianças são: avaliação antropométrica, saúde bucal, oftalmológica, verificação da situação vacinal. As optativas são: avaliação auditiva e de alterações da linguagem oral. Para os adolescentes, há ainda a temática optativa de identificar possíveis sinais de agravos de doenças em eliminação (BRASIL, 2015).

As estratégias de promoção e prevenção da saúde para crianças, no programa saúde nas escolas, são: alimentação saudável, cultura da paz e direitos humanos, saúde mental nas escolas. Como optativas: violência e acidentes, saúde mental nas escolas (direcionada aos familiares), estratégia NutriSUS (fortificação da alimentação com micronutrientes em pó, práticas corporais de atividade física e lazer, saúde ambiental e desenvolvimento sustentável. Para os adolescentes, há ainda: sexualidade, reprodução e Infecções Sexualmente Transmissíveis, uso de álcool, tabagismo e outras drogas; e como optativa, administração de conflitos nas escolas (BRASIL, 2015).

Nos artigos selecionados, as ações e práticas educativas dos enfermeiros ou estudantes de enfermagem para atingirem seus objetivos foram: a utilização de ação educativa crítica, conversas e entrevistas, oficinas pedagógicas, antropometria e práticas lúdicas. 
As ações educativas a serem desenvolvidas nas escolas, com crianças e adolescentes, segundo o Ministério da Saúde (2015), são: avaliação antropométrica; situação vacinal; saúde bucal; saúde ocular; saúde auditiva; desenvolvimento de linguagem; reconhecimento de vestígios de patologias predominantes na região e realizar monitorização e providências; alimentação saudável; educação alimentar; cultura de paz e dos diretos humanos; saúde mental; prevenção da saúde; prevenção de acidentes; saúde ambiental; práticas corporais exercícios: disponibilizar atividade física e de lazer.

As práticas desenvolvidas com os escolares têm ocorrido de forma gratificante, com resultados positivos relacionados à saúde do escolar e seus familiares. As ações educativas desenvolvidas, em países de baixa renda são: remoção de parasitas, alimentação nas escolas, imunização, antropometria, triagem de visual e auditiva, saneamento e higiene, atividade física, alimentação saudável, saúde bucal e tabagismo (WORLD HEALTH ORGANIZATION, 2017).

Entre as funções do enfermeiro atuante nas escolas, destacam-se: programar e efetuar as ações de promoção e prevenção à saúde do escolar (antropometria, IMC, alimentação saudável, doenças crônicas não transmissíveis), verificar riscos a saúde e encaminhar para atendimento, organizar atividades de educação permanente para a saúde (BRASIL, 2009).

O enfermeiro atuando nas escolas desenvolve ações de educação, promoção e prevenção em saúde no ambiente escolar, favorecendo a diminuição nas taxas de morbidade e mortalidade, pela intervenção nos fatores de risco de diversas doenças.

\section{ConClusão}

A presente revisão integrativa permitiu selecionar na literatura estudos sobre a temática, no qual predominaram as intervenções de características educativas para as crianças e relacionadas à prevenção de doenças crônicas não transmissíveis no contexto da saúde escolar.

As ações e práticas educativas dos enfermeiros ou estudantes de enfermagem foram concretizadas através da utilização de ações educativas, conversas e entrevistas, oficinas pedagógicas, antropometria e práticas lúdicas.

Como limitações desse estudo, reconhecemos que dizem respeito aos próprios critérios de inclusão e exclusão dos estudos, além de ter se restringido o fato da busca em apenas três bases de dados, o que retrata apenas um recorte de estudos da área. Recomenda-se a realização de outros estudos nacionais com o intuito de aprofundar o debate sobre a temática, fortalecendo o trabalho do profissional enfermeiro e quebras de paradigmas de práticas no contexto da saúde escolar.

Conclui-se que este tema ainda é incipiente em termos de publicações sobre a prática educativas do enfermeiro nas escolas, atuando diretamente com crianças e adolescentes, tendo em vista a importância do profissional enfermeiro atuando nas escolas para a promoção e prevenção em saúde.

\section{REFERÊNCIAS}

AGENCY FOR HEALTHCARE RESEARCH AND QUALITY. Content last reviewed. Agency for Healthcare Research and Quality, Rockville, MD. 2017. Disponível em: <http://www.ahrq.gov/cpi/about/index. html> Acesso em: 12 jan. 2019.

AMERICAN ACADEMY OF PEDIATRICS. Role of the School Nurse in Providing School Health Services. AMERICAN ACADEMY OF PEDIATRICS, v.121, n. 5, p. 1052-8, May 2008.

BRANDÃO NETO, W. et al. Intervenção educativa sobre violência com adolescentes: possibilidade para a enfermagem no contexto escolar. Esc. Anna Nery, Rio de Janeiro, v. 18, n. 2, p. 195-201, Jun. 2014. 
Joia et al.

BRASIL. Caderno do gestor do PSE: Ministério da Saúde, Ministério da Educação. Brasília: Ministério da Saúde, 2015.

BRASIL. Ministério da Saúde. Secretaria de Atenção à Saúde. Departamento de Atenção Básica. Saúde na escola / Ministério da Saúde, Secretaria de Atenção à Saúde, Departamento de Atenção Básica. - Brasília: Ministério da Saúde, 2009.

CASEMIRO, J. P.; FONSECA, A. B. C.; SECCO, F. V. M. Promover saúde na escola: reflexões a partir de uma revisão sobre saúde escolar na América Latina. Ciência \& Saúde Coletiva [online]. 2014, v. 19, n. 03, p. 829-40. Disponível em: <https://doi.org/10.1590/1413-81232014193.00442013>. Acesso em: 10 mar.19.

COSTA, G. M. C. et al. Promoção de saúde nas escolas na perspectiva de professores do ensino fundamental. Revista Eletrônica de Enfermagem, Goiás, v.15, n.2, p.506-15, abr./jun. 2013. Disponível em: <https://www.fen.ufg.br/fen_revista/v15/n2/pdf/v15n2a25.pdf >. Acesso em 30 abr. 19.

COSTA, G. M.; FIGUEIREDO, R. C.; RIBEIRO, M. S. A importância do enfermeiro junto ao PSE nas ações de educação em saúde em uma escola municipal de Gurupi-TO. Rev Científica ITPAC, Araguaína, v.6, n.2, abr. 2013. Disponível em: < http://www.itpac.br/arquivos/Revista/62/6.pdf_>. Acesso em 30 abr. 19.

FERNANDINO, M. N. Enfermeria escolar: una revisión bibliográfica. Tortosa: Universitat Rovira I Virgili - Facultat d'Infermeria, 2016.

FERREIRA, A. D. S., et al. A história da Educação em Saúde e seus modelos de prática impostos à sociedade. Diversitas Journal, Santana do Ipanema, v.1, n.1, p.48-54, jan./abr.2016. Disponível em: <http:// www.kentron.ifal.edu.br/index.php/diversitas_journal/article/view/379 >. Acesso em 30 abr. 19.

FRIEDRICH, B.V.; PIGOTT, T. A Practitioner-Based Asthma Intervention Program With. Journal of Pediatric Health Care, P. 163-6, May/June 2005.

GANONG, L.H. Integrative reviews of nursing research. Res Nurs Health, v. 10, n.1, p. 1-11, 1987. Disponível em: <https://www.ncbi.nlm.nih.gov/pubmed/3644366>. Acesso em: 08 de out. 2019.

GIJSEN, L. I. P. S.; KAISER, D. E. Enfermagem e educação em saúde em escolas no Brasil: revisão integrativa da literatura. Ciência, cuidado e saúde, Maringá. Vol. 12, n. 4, p. 813-821, out./dez. 2013. Disponível em: <http://periodicos.uem.br/ojs/index.php/CiencCuidSaude/article/view/17618 >. Acesso em 30 abr. 19.

JOHNSTON, J.C.T. et al. A Growth Status Measurement Pilot in Four Calgary Area Schools: Perceptions of Grade 5 Students and Their Parents. The Journal of School Nursing, v. 27, n. 1, p. 61-9, February 2011.

LEHNA, C. et al. Nursing students practice primare fire prevention. B u r n s, v. 3 9, p. 1277 - 1284 , 2013. Disponível em: <www.sciencedirect.com>. Acesso em: 08 de nov. 2019.

MENDES, K.D.S.; SILVEIRA, R.C.C.P.; GALVÃO, C.M. Revisão integrativa: método de pesquisa para a incorporação de evidências na saúde e na enfermagem. Texto Contexto Enferm., 2008, v. 17, n.4, p.75864. Disponível em:< http://dx.doi.org/10.1590/S0104-07072008000400018>. Acesso em: 08 de nov. 2019. 
NORTHRUP, K. L. et al. L.I.F.E.: A School-Based Heart-Health Screening and Intervention Program. The Journal of School Nursing, v. 24, n.1, p.28-36, February 2008.

OLIVEIRA, M. A. F. C.; BUENO, S. M. V. Comunicação educativa do enfermeiro na promoção da saúde sexual do escolar. Rev. Latino-Am. Enfermagem, Ribeirão Preto, v. 5, n. 3, p. 71-81, jul. 1997. Disponível em <http://www.scielo.br/scielo.php?script=sci_arttext\&pid=S0104=11691997000300011-\&lng=pt\&nrmiso >. Acesso em: 07 jul. 2018.

ORGANIZAÇÃO PAN-AMERICANA DA SAÚDE. Ministério da Saúde. Saúde e sexualidade de adolescentes. Construindo equidade no SUS. Brasília, DF: OPAS, MS, 2017.

RASCHE, A. S.; SANTOS, M. S. S. Enfermagem escolar e sua especialização: uma nova ou antiga atividade. Revista Brasileira de Enfermagem; Brasília, v. 66, n. 4, p. 607-610, Jul/Aug 2013. Disponível em: <http:// www.scielo.br/scielo.php?script=sci_arttext\&pid=S0034-71672013000400022>. Acesso em: 10 mar. 19.

SKYBO, T. A.; WENGER, N. R. A School-Based Intervention to Teach Third Grade Children ahout Continuing Education the Prevention of Heart Disease. Pediatric Nursing, V. 22, N. 3, May-June 2002

URSI, E.S. Prevenção de lesões de pele no perioperatório: revisão integrativa da literatura. Universidade de São Paulo, Ribeirão Preto, 2005. Disponível em: <http://doi.org //10.11606/D.22.2005.tde-18072005095456>. Acesso em: 12 abr. 2019.

WORLD HEALTH ORGANIZATION. Assessing and managing children at primary health-care facilities to prevent overweight and obesity in the context of the double burden of malnutrition. Updates for the Integrated Management of Childhood Illness (IMCI). Geneva: World Health Organization; 2017. Disponivel em: <http://apps.WORLD HEALTH ORGANIZATION.int/iris/bitstream/ handle/10665/259133/9789241550123-eng.pdf? sequence=1>. Acesso em: 24 nov. 2019.

WORLD HEALTH ORGANIZATION. Global school health initiatives: achieving health and education outcomes. Report of a meeting, Bangkok, Thailand, 23-25 November 2015. Geneva: World Health Organization; 2017. Disponivel em: <http://apps.WORLD HEALTH ORGANIZATION.int/iris/bitstream/ handle/10665/259813/WORLD HEALTH ORGANIZATION-NMH-PND-17.7-eng.pdf;jsessionid=32BDC79FF3CD1400250E309735709C8D? sequence=1>. Acesso em: 05 dez. 2018.

WORLD HEALTH ORGANIZATION. Relatório Mundial Sobre a Prevenção da Violência 2014. São Paulo: Núcleo de Estudos da Violência da Universidade de São Paulo (tradução), 2015. Disponivel em: < apps.WORLD HEALTH ORGANIZATION.int/iris/bitstream/10665/145086/5/9789241564793_por. pdf>. Acesso em: 24 nov. 2018.

WORLD HEALTH ORGANIZATION. World report on child injury prevention. Geneva: World Health Organization (WORLD HEALTH ORGANIZATION); 2008. Disponível em: <http://apps.WORLD HEALTH ORGANIZATION.int/iris/bitstream/handle/10665/43851/9789241563574_;jsessionid=3D7D4921E91B7BB1C81B58AF918B07A0? sequence=1>. Acesso em: 24 nov. 2019.

VASCONCELOS, V. M. et al. Educação em saúde na escola: estratégia em enfermagem na prevenção da 
Joia et al.

desnutrição infantil. Ciência Cuidado e Saúde, v. 7, n. 3, p. 355-362, Jul/Set 2008. Disponível em: <http:// www.periodicos.uem.br/ojs/index.php/CiencCuidSaude/article/view/6508/3862>. Acesso em: 07 Jul 2019. 